\title{
The postoperative neutrophil-to-lymphocyte ratio and changes in this ratio predict survival after the complete resection of stage I non-small cell lung cancer
}

\author{
This article was published in the following Dove Press journal: \\ OncoTargets and Therapy \\ 21 October 2016 \\ Number of times this article has been viewed
}

\section{Feng Jin ${ }^{1,2}$ \\ Anqin $\mathrm{Han}^{2}$ \\ Fang $\mathrm{Shi}^{2}$ \\ Li Kong ${ }^{2}$ \\ Jinming $\mathrm{Yu}^{2}$}

'School of Medicine and Life Sciences, University of Jinan-Shandong Academy of Medical Sciences, ${ }^{2}$ Department of Radiation Oncology, Shandong Cancer Hospital Affiliated to Shandong University, Shandong Academy of Medical Sciences, Jinan, People's Republic of China
Correspondence: Jinming Yu Department of Radiation Oncology, Shandong Cancer Hospital Affiliated to Shandong University, Shandong Academy of Medical Sciences, Jiyan Road 440, Jinan 250I I7, Shandong, People's Republic of China

Tel +86 53। 87984729

Fax +86 53। 87984079

Email sdyujinming@sina.com
Purpose: Although numerous studies have demonstrated associations between the preoperative neutrophil-to-lymphocyte ratio (NLR) and long-term outcomes in patients with non-small cell lung cancer (NSCLC), the prognostic significance of postoperative NLR and change in NLR ( $\triangle \mathrm{NLR})$ is unknown for patients who underwent complete resection of stage I NSCLC. The aim of this retrospective study was to evaluate the prognostic significance of postoperative NLR and $\triangle$ NLR in 123 patients with stage I NSCLC.

Patients and methods: This retrospective study included preoperative and postoperative data of 123 patients who underwent surgical resection for stage I NSCLC. The relationship between disease-free survival (DFS), overall survival (OS), and clinicopathological factors, including NLR, lymphocyte-to-monocyte ratio (LMR), platelet-to-lymphocyte ratio, and their changes, was analyzed using both univariate Kaplan-Meier and multivariate Cox regression methods.

Results: The 5-year DFS and OS rates in our cohort were $60.16 \%$ and $67.48 \%$, respectively. Univariate analysis revealed that age $(P=0.045)$, smoking status $(P=0.033)$, preoperative NLR $(P=0.032)$, postoperative NLR $(P<0.001), \Delta$ NLR $(P=0.004)$, and change in LMR $(\triangle \mathrm{LMR})(P=0.025)$ were significant predictors of DFS and that age $(P=0.039)$, smoking status $(P=0.042)$, postoperative NLR $(P<0.001), \Delta$ NLR $(P=0.004)$, and $\Delta$ LMR $(P=0.011)$ were independent predictors of OS. Multivariate analysis confirmed that postoperative NLR (hazard ratio $[\mathrm{HR}]=2.435, P=0.001)$ and $\Delta \mathrm{NLR}(\mathrm{HR}=2.103, P=0.012)$ were independent predictors of DFS and that postoperative NLR $(\mathrm{HR}=2.747, P=0.001)$ and $\triangle \mathrm{NLR}(\mathrm{HR}=2.052, P=0.018)$ were significant prognostic factors of OS.

Conclusion: Our study reported for the first time that postoperative NLR and $\triangle$ NLR - but not preoperative NLR - were independent prognostic factors of DFS and OS in patients with stage I NSCLC who underwent complete resection. This easily available biomarker might be helpful in individual risk assessment.

Keywords: neutrophil-to-lymphocyte ratio, non-small-cell lung cancer, stage I, prognosis, surgery

\section{Introduction}

Lung cancer is the second most common newly diagnosed cancer and one of the leading causes of cancer-related death among both men and women worldwide. ${ }^{1}$ Surgical resection is the treatment of choice for patients with early-stage non-small cell lung cancer (NSCLC). ${ }^{2}$ Despite aggressive surgical therapy, $\sim 30 \%-40 \%$ of patients with 
stage I NSCLC develop tumor recurrence in a short time. ${ }^{3}$ Therefore, a reliable and inexpensive biomarker is needed to predict survival in patients with NSCLC and identify the subgroups of patients who will benefit from aggressive postoperative treatment.

Recently, a systemic inflammatory marker for prognosis, the neutrophil-to-lymphocyte ratio (NLR), has received considerable interest because it is simple, convenient, and inexpensive. ${ }^{4}$ Although numerous studies have demonstrated associations between preoperative NLR and outcomes in primary operable patients with NSCLC, ${ }^{5-8}$ other studies have concluded the opposite. ${ }^{9,10}$ Moreover, some studies found that postoperative NLR could predict the survival of patients with hepatocellular carcinoma, ${ }^{11}$ colorectal cancer, ${ }^{12}$ and bladder cancer after complete resection. ${ }^{13}$ These results suggest that postoperative NLR might provide a more precise reflection of the balance between tumor inflammatory response and host immune response.

The change in NLR ( $\Delta$ NLR) represents the balance between protumor inflammatory response and antitumor immune response after the surgical removal of the tumor, which may affect prognosis. ${ }^{14}$ Some studies have found that $\triangle$ NLR is a useful prognostic predictor in patients with hepatocellular carcinoma. ${ }^{14,15}$ However, the prognostic significance of postoperative NLR and $\triangle N L R$ is unknown for patients undergoing complete resection of stage I NSCLC.

Accordingly, the aim of this retrospective study was to evaluate the prognostic significance of postoperative NLR and $\triangle N L R$ in patients with stage I NSCLCs that were treated with complete resection.

\section{Patients and methods}

\section{Patient cohort}

This was a retrospective study of patients with stage I NSCLC who underwent complete pulmonary resection (lobectomy or pneumonectomy) and systematic dissection of the hilar and mediastinal lymph nodes between January 2007 and December 2010 at the Shandong Cancer Hospital and Institute (Jinan, China). The exclusion criteria were as follows: chemotherapy or radiotherapy before the surgery; recurrence; clinical evidence of preoperative or postoperative infection or other bone marrow disorders; intraoperative or postoperative complications; recent steroid therapy; intraoperative or postoperative blood transfusion; hematological or autoimmune disease; history of another type of cancer; and a second primary cancer diagnosed within 5 years. All patients received chemotherapy with or without radiotherapy after recurrence. After applying the criteria mentioned earlier, 123 patients with stage I NSCLC were recruited for this study. All of the clinicopathological data were retrieved from medical records. The preoperative evaluation included a detailed clinical history, physical examination, blood chemistry analysis, flexible bronchoscopy, chest and upper abdominal computed tomography, radionuclide bone scan, and brain magnetic resonance imaging. The histological diagnosis of NSCLC was based on the criteria of the revised World Health Organization classification of lung tumors and tumor staging (7th edition) and the TNM classification ( $\mathrm{T}$, tumor size; $\mathrm{N}$, involvement of lymph nodes; M, distant metastasis). ${ }^{16,17}$ This study was approved by the Ethics Committee of Shandong Cancer Hospital and Institute, Jinan, China, and written informed consent was obtained from each participant in accordance with institutional guidelines. All treatments were carried out in accordance with the approved guidelines and regulations.

All 123 patients were regularly followed up at 3-month intervals for the first 2 years after surgery, every 6 months during the following year, and annually thereafter. The complete follow-up information was recorded, including the findings of physical examinations, chest computed tomography, abdominal ultrasound, and blood analyses (including serum tumor markers). The last date of follow-up occurred at the end of October 2015.

\section{Definition of inflammatory markers and survival}

Peripheral venous blood samples were collected between 8 am and 10 am within 1 week before surgery and at least 1 month after surgery. The NLR was determined from the differential count by dividing the absolute neutrophil count by the absolute lymphocyte count. $\triangle \mathrm{NLR}$ was calculated as postoperative NLR minus preoperative NLR. The lymphocyte-tomonocyte ratio (LMR), platelet-to-lymphocyte ratio (PLR), and their changes (change in LMR $[\Delta \mathrm{LMR}]$ and change in PLR $[\triangle \mathrm{PLR}]$ ) were calculated in a similar manner. The overall survival (OS) time was defined as the interval between the operation and death or the last follow-up. The disease-free survival (DFS) time was defined as the interval between the operation and the first incidence of detectable recurrence. Patients who remained disease free or alive were censored at the final follow-up. DFS and OS were used to demonstrate the prognostic values of inflammatory markers.

\section{Statistical analysis}

All statistical analyses were conducted using SPSS for Windows, version 20.0 (IBM Corporation, Armonk, NY, USA). Pretreatment and posttreatment inflammatory markers, including NLR, LMR, PLR, and their changes ( $\triangle \mathrm{NLR}, \triangle \mathrm{LMR}$, and $\triangle \mathrm{PLR})$, were entered into the univariate and multivariate 
analyses to identify the associations with DFS and OS. To determine the optimal cutoff value for each inflammatory marker, a receiver operating characteristic (ROC) curve was generated, and the cutoff value was defined as the point on the curve that was closest to the upper left-hand corner of the plot. According to previously published study, ${ }^{18}$ the median age of the study cohort was used as a cutoff value for age. DFS and OS were summarized descriptively, and the accompanying two-sided 95\% CIs were also calculated. The DFS and OS rates were estimated using the Kaplan-Meier method, and log-rank tests were used for the univariate analysis. Variables that showed significant associations in the univariate analysis $(P<0.05)$ were included in a multivariate stepwise backward Cox regression model to validate their independent prognostic values. Differences were assumed to be significant when a $P$-value of $<0.05$ was achieved.

\section{Results}

\section{Baseline characteristics}

A total of 123 consecutive patients with complete medical information and follow-up data were included in the analysis. The baseline characteristics of the patients are presented in

Table 1 . The study cohort included 94 men (76.42\%) and

Table I Baseline characteristics of the 123 patients

\begin{tabular}{|c|c|c|}
\hline Clinical feature & $\mathbf{N}$ & Percentage \\
\hline \multicolumn{3}{|l|}{ Sex } \\
\hline Male & 94 & 76.42 \\
\hline Female & 29 & 23.58 \\
\hline Age (median), years & & $(62)$ \\
\hline$\leq 62$ & 62 & 50.41 \\
\hline$>62$ & 61 & 49.59 \\
\hline \multicolumn{3}{|l|}{ ECOG scores } \\
\hline 0 & 79 & 64.23 \\
\hline I & 39 & 31.71 \\
\hline$\geq 2$ & 5 & 4.06 \\
\hline \multicolumn{3}{|l|}{ Smoking status } \\
\hline Yes & 73 & 59.35 \\
\hline No & 50 & 40.65 \\
\hline \multicolumn{3}{|l|}{ Histological subtype } \\
\hline Adenocarcinoma & 60 & 48.78 \\
\hline Squamous carcinoma & 49 & 39.84 \\
\hline Others & 14 & 11.38 \\
\hline \multicolumn{3}{|l|}{ Differentiation } \\
\hline Well/moderate & 98 & 79.67 \\
\hline Poor & 25 & 20.33 \\
\hline \multicolumn{3}{|l|}{ Pathological stage } \\
\hline Stage IA & 63 & 51.22 \\
\hline Stage IB & 60 & 48.78 \\
\hline \multicolumn{3}{|l|}{ Tumor location } \\
\hline Left & 50 & 40.65 \\
\hline Right & 73 & 59.35 \\
\hline \multicolumn{3}{|l|}{ Surgical procedures } \\
\hline Lobectomy & 104 & 84.55 \\
\hline Pneumonectomy & 19 & 15.45 \\
\hline
\end{tabular}

Abbreviation: ECOG, Eastern Cooperative Oncology Group.
29 women $(23.58 \%$ ), with a median age of 62 years (range: 39-81 years). The numbers of patients with Eastern Cooperative Oncology Group scores of 0 and 1 (considered good) were $79(64.23 \%)$ and $39(31.71 \%)$, respectively. Seventythree $(59.35 \%)$ patients had a history of smoking. Sixty (60\%) patients had adenocarcinoma, 49 (39.84\%) patients had squamous cell carcinoma, and 14 (11.38\%) patients had other histologies. Ninety-eight (79.67\%) patients had a well or moderately differentiated histology, and 63 (51.22\%) patients were of stage IA disease. Of the 123 patients, 104 (84.55\%) patients underwent a lobectomy and 19 (15.45\%) patients underwent a pneumonectomy. The median follow-up time was 69 months (range: 9-107 months). The details of treatment after recurrence are as follows: all relapsed patients received more than four cycles of platinum-based chemotherapy, 38 patients received thoracic radiotherapy; 20 patients received brain metastases radiotherapy, and 11 patients received bone metastases radiotherapy. There were 49 patients of recurrence, 40 deaths, and 83 patients who were alive at the time of the final follow-up. The 5-year DFS and OS rates were $60.16 \%$ and $67.48 \%$, respectively. The patients exhibited a median preoperative NLR of 2.3 (range: 0.87-10.07), a median postoperative NLR of 3.94 (range: $0.54-15.11$ ), and a median $\triangle$ NLR of 1.9 (range: -7.97 to 12.86). All of the inflammation markers that were calculated from blood cell counts are summarized in Table 2.

\section{ROC curves for predictions of recurrence}

This study performed ROC analyses to select appropriate cutoff values for the inflammatory markers. The cutoff value was 2.5 for preoperative NLR, with an area under the curve (AUC) of 0.614. Preoperative NLR had a sensitivity of $65.3 \%$

Table 2 Inflammation markers of the 123 patients

\begin{tabular}{ll}
\hline Variable & Median (range) \\
\hline NLR & \\
$\quad$ Preoperative NLR & $22.3(0.87$ to I0.07) \\
Postoperative NLR & $33.94(0.54$ to I5.I I) \\
$\Delta$ NLR & $1.9(-7.97$ to I2.86) \\
LMR & \\
$\quad$ Preoperative LMR & $33.7(0.95$ to I5.25) \\
Postoperative LMR & $2.16(0.6$ I to 25.5$)$ \\
$\Delta$ LMR & $-1.53(-13.42$ to 21.06$)$ \\
PLR & \\
$\quad$ Preoperative PLR & $108.26(31.32$ to 270.53$)$ \\
Postoperative PLR & $1,161.67(4 I .95$ to 608.74$)$ \\
$\Delta$ PLR & $557.85(-136.09$ to 369.58$)$ \\
\hline
\end{tabular}

Abbreviations: $L M R$, lymphocyte-to-monocyte ratio; $\triangle L M R$, change in $L M R ; N L R$, neutrophil-to-lymphocyte ratio; $\triangle N L R$, change in NLR; PLR, platelet-to-lymphocyte ratio; $\triangle \mathrm{PLR}$, change in PLR. 


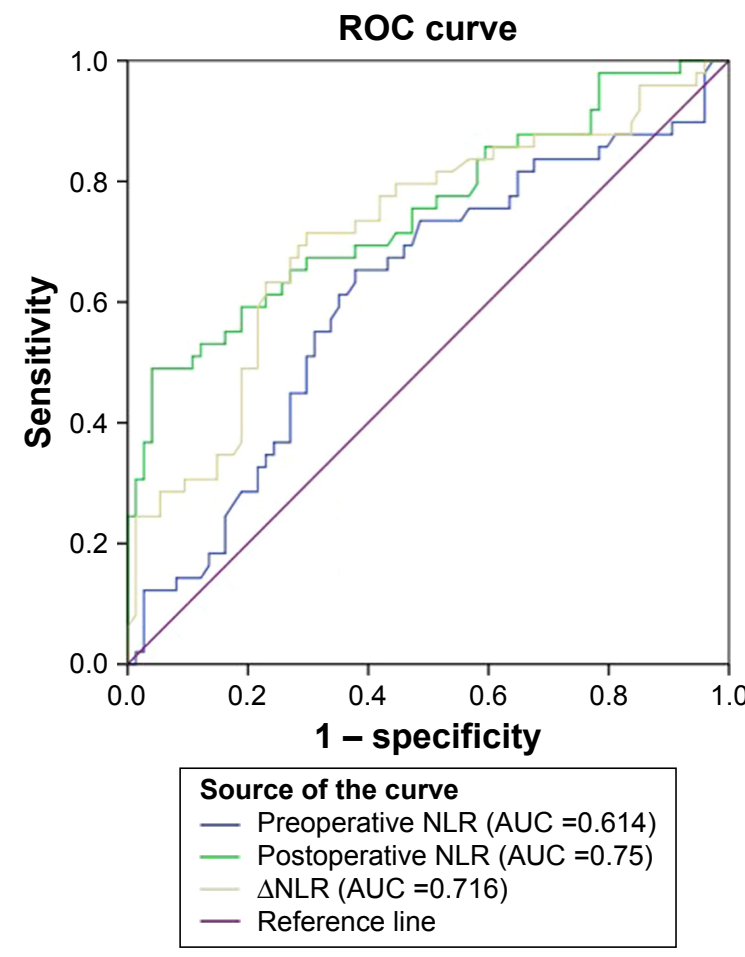

Figure I ROC curves for recurrence prediction.

Abbreviations: AUC, area under the curve; NLR, neutrophil-to-lymphocyte ratio; $\triangle N L R$, change in NLR; ROC, receiver operating characteristic. and a specificity of $62.2 \%$ for predicting recurrence. The best cutoff values for postoperative NLR and $\triangle$ NLR were 3.9 and 1.5, respectively, with AUCs of 0.75 (sensitivity: 67.3\%, specificity: $70.3 \%$ ) and 0.716 (sensitivity: $71.4 \%$, specificity: 69.3\%), respectively (Figure 1). The AUCs for preoperative LMR, postoperative LMR, $\triangle \mathrm{LMR}$, preoperative PLR, postoperative PLR, and $\triangle$ PLR were $0.534,0.467$, $0.428,0.559,0.358$, and 0.445 , respectively. The selected cutoff values for preoperative LMR, postoperative LMR, $\triangle \mathrm{LMR}$, preoperative PLR, postoperative PLR, and $\triangle \mathrm{PLR}$ were $2.9,2.6,-1.5,100,144$, and 24 , respectively.

\section{Inflammatory markers and survival}

Univariate analysis revealed that age $(P=0.045)$, smoking status ( $P=0.033)$, preoperative NLR $(P=0.032)$, postoperative NLR $(P<0.001), \triangle \mathrm{NLR}(P=0.004)$, and $\triangle \mathrm{LMR}(P=0.025)$ were significant predictors of DFS (Tables 3 and 4 ) and that age $(P=0.039)$, smoking status $(P=0.042)$, postoperative NLR $(P<0.001), \Delta$ NLR $(P=0.004)$, and $\triangle \mathrm{LMR}(P=0.011)$ were prognostic factors of OS (Tables 3 and 4). Preoperative LMR, postoperative LMR, preoperative PLR, postoperative PLR, and $\triangle \mathrm{PLR}$ did not predict DFS or OS after surgery.

Table 3 Univariate Kaplan-Meier analysis of DFS and OS according to baseline characteristics

\begin{tabular}{|c|c|c|c|c|c|c|}
\hline \multirow[t]{2}{*}{ Factor } & \multicolumn{3}{|l|}{ DFS } & \multicolumn{3}{|l|}{ OS } \\
\hline & Median (months) & $95 \% \mathrm{Cl}$ & $P$-value & Median (months) & $95 \% \mathrm{Cl}$ & $P$-value \\
\hline Sex & & & 0.323 & & & 0.376 \\
\hline Male & 63 & $56.955-69.045$ & & 66 & $60.884-71.116$ & \\
\hline Female & 69 & $67.022-70.978$ & & 69 & $66.74-71.26$ & \\
\hline Age, years & & & 0.045 & & & 0.039 \\
\hline$\leq 62$ & 69 & 63.599-74.40I & & 71 & $66.202-75.798$ & \\
\hline$>62$ & 61 & $50.418-71.582$ & & 66 & $59.023-72.977$ & \\
\hline ECOG & & & 0.413 & & & 0.51 \\
\hline 0 & 68 & $62.741-73.259$ & & 69 & $65.495-72.505$ & \\
\hline 1 & 66 & $60.822-71.178$ & & 66 & $61.469-70.531$ & \\
\hline$\geq 2$ & 29 & $0-65.5$ & & 54 & $30.382-77.618$ & \\
\hline Smoking status & & & 0.033 & & & 0.042 \\
\hline Yes & 62 & $50.363-73.637$ & & 66 & $59.348-72.652$ & \\
\hline No & 69 & $65.904-75.096$ & & 69 & $65.008-74.992$ & \\
\hline Histological subtype & & & 0.571 & & & 0.626 \\
\hline Adenocarcinoma & 66 & $60.581-71.419$ & & 67 & $63.628-70.372$ & \\
\hline Squamous & 67 & $54.655-79.345$ & & 69 & $63.522-74.478$ & \\
\hline Others & 69 & $67.185-70.815$ & & 69 & $54.333-83.667$ & \\
\hline Differentiation & & & 0.871 & & & 0.967 \\
\hline Well/moderate & 67 & $64.016-69.984$ & & 69 & $66.105-71.895$ & \\
\hline Poor & 61 & $40.716-81.284$ & & 67 & $57.208-76.792$ & \\
\hline Pathological stage & & & 0.661 & & & 0.759 \\
\hline Stage IA & 67 & $59.413-74.587$ & & 67 & 59.409-74.59| & \\
\hline Stage IB & 67 & $63.543-70.457$ & & 69 & $65.687-72.313$ & \\
\hline Tumor location & & & 0.094 & & & 0.144 \\
\hline Left & 69 & $66.376-71.624$ & & 70 & $67.006-72.994$ & \\
\hline Right & 63 & $57.458-68.542$ & & 66 & $61.524-70.476$ & \\
\hline Surgical procedures & & & 0.138 & & & 0.133 \\
\hline Lobectomy & 67 & $63.255-70.745$ & & 67 & $64.145-69.855$ & \\
\hline Pneumonectomy & 69 & $54.781-83.219$ & & 69 & $64.734-73.266$ & \\
\hline
\end{tabular}

Abbreviations: Cl, confidence interval; DFS, disease-free survival; ECOG, Eastern Cooperative Oncology Group; OS, overall survival. 
Table 4 Univariate Kaplan-Meier analysis of DFS and OS according to inflammatory markers

\begin{tabular}{|c|c|c|c|c|c|c|}
\hline \multirow{2}{*}{$\begin{array}{l}\text { Inflammatory } \\
\text { markers }\end{array}$} & \multicolumn{3}{|l|}{ DFS } & \multicolumn{3}{|l|}{ os } \\
\hline & Median (months) & $95 \% \mathrm{Cl}$ & $P$-value & Median (months) & $95 \% \mathrm{Cl}$ & $P$-value \\
\hline Preoperative NLR & & & 0.032 & & & 0.061 \\
\hline$\leq 2.5$ & 70 & $67.838-72.162$ & & 70 & $67.158-72.842$ & \\
\hline$>2.5$ & 49 & $25.75-72.25$ & & 60 & $50.489-69.5 \mathrm{II}$ & \\
\hline Postoperative NLR & & & $<0.00 \mathrm{I}$ & & & $<0.001$ \\
\hline$\leq 3.9$ & 70 & $67.374-72.626$ & & 71 & 68.299-73.70I & \\
\hline$>3.9$ & 40 & $24.932-55.068$ & & 56 & $40.546-71.454$ & \\
\hline$\Delta \mathrm{NLR}$ & & & 0.004 & & & 0.004 \\
\hline$\leq 1.5$ & 72 & $68.801-75.199$ & & 72 & $69.27-76.73$ & \\
\hline$>1.5$ & 60 & $48.161-71.839$ & & 61 & $56.69 \mid-65.309$ & \\
\hline Preoperative LMR & & & 0.311 & & & 0.566 \\
\hline$\leq 2.9$ & 66 & $61.851-70.149$ & & 67 & 64.329-69.67I & \\
\hline$>2.9$ & 71 & $64.07-77.93$ & & 70 & $61.684-78.316$ & \\
\hline Postoperative LMR & & & 0.103 & & & 0.116 \\
\hline$\leq 2.6$ & 65 & 58.903-7I.097 & & 69 & 60.919-71.08I & \\
\hline$>2.6$ & 69 & $65.285-72.715$ & & 69 & $63.583-74.417$ & \\
\hline$\Delta \mathrm{LMR}$ & & & 0.025 & & & 0.011 \\
\hline$\leq-1.5$ & 65 & $60.217-69.783$ & & 66 & 60.539-7I.46I & \\
\hline$>-1.5$ & 70 & $64.856-75.144$ & & 71 & $65.858-76.142$ & \\
\hline Preoperative PLR & & & 0.244 & & & 0.291 \\
\hline$\leq 100$ & 69 & $65.991-72.009$ & & 69 & $65.333-72.667$ & \\
\hline$>100$ & 66 & $57.728-74.272$ & & 66 & $61.156-70.844$ & \\
\hline Postoperative PLR & & & 0.291 & & & 0.268 \\
\hline$\leq \mathrm{I} 44$ & 69 & $65.469-72.531$ & & 69 & $66.646-71.354$ & \\
\hline$>144$ & 63 & $56.583-69.417$ & & 66 & $60.288-71.712$ & \\
\hline$\Delta \mathrm{PLR}$ & & & 0.849 & & & 0.931 \\
\hline$\leq 24$ & 69 & $65.5 \mid 3-72.487$ & & 69 & $66.724-71.276$ & \\
\hline$>24$ & 63 & $57.948-68.052$ & & 66 & $59.713-72.287$ & \\
\hline
\end{tabular}

Abbreviations: $\mathrm{Cl}$, confidence interval; DFS, disease-free survival; LMR, lymphocyte-to-monocyte ratio; $\Delta \mathrm{LMR}$, change in LMR; NLR, neutrophil-to-lymphocyte ratio; $\Delta \mathrm{NLR}$, change in NLR; OS, overall survival; PLR, platelet-to-lymphocyte ratio; $\triangle \mathrm{PLR}$, change in PLR.

Multivariate analysis confirmed that postoperative NLR (hazard ratio $[\mathrm{HR}]=2.435,95 \% \mathrm{CI}: 1.526-4.322, P=0.001$ ) and $\triangle \mathrm{NLR}(\mathrm{HR}=2.103,95 \% \mathrm{CI}: 1.332-3.883, P=0.012)$ were independent predictors of DFS and that postoperative NLR (HR $=2.747,95 \%$ CI: 1.668-4.408, $P=0.001)$ and $\triangle$ NLR (HR $=2.052$, 95\% CI: $1.225-3.468, P=0.018$ ) were significant prognostic factors of OS (Table 5).

The Kaplan-Meier method was used to estimate DFS and OS curves, as stratified according to postoperative NLR and $\triangle$ NLR values (Figures 2 and 3). Differences in survival were evaluated using the log-rank test, which showed clear distinctions for all strata (DFS analyses: $P<0.001$ for postoperative NLR and $P=0.004$ for $\triangle \mathrm{NLR}$; OS analyses: $P<0.001$ for postoperative NLR and $P=0.004$ for $\Delta$ NLR).

\section{Discussion}

Several previous studies have suggested that preoperative inflammatory markers, such as NLR, LMR, and PLR, are useful

Table 5 Multivariate Cox regression analysis of DFS and OS in patients with stage I NSCLC

\begin{tabular}{|c|c|c|c|c|}
\hline \multirow[t]{2}{*}{ Factor } & \multicolumn{2}{|l|}{ DFS } & \multicolumn{2}{|l|}{ OS } \\
\hline & HR (95\% CI) & $P$-value & HR (95\% CI) & $P$-value \\
\hline Age $(\leq 62 />62$, years $)$ & $\mathrm{I} .457(0.724-2.472)$ & 0.143 & I.20I (0.534-I.945) & 0.223 \\
\hline Smoking (yes/no) & I.I I3 (0.693-I.859) & 0.645 & $0.887(0.367-\mathrm{I} .458)$ & 0.826 \\
\hline Preoperative NLR $(\leq 2.5 />2.5)$ & $1.798(1.127-2.869)$ & 0.074 & & \\
\hline Postoperative NLR $(\leq 3.9 />3.9)$ & $2.435(1.526-4.322)$ & 0.001 & $2.747(1.668-4.408)$ & 0.001 \\
\hline$\Delta \mathrm{NLR}(\leq \mathrm{I} .5 />1.5)$ & $2.103(1.332-3.883)$ & 0.012 & $2.052(1.225-3.468)$ & 0.018 \\
\hline$\Delta \operatorname{LMR}(\leq-|.5 />-| .5)$ & $\mathrm{I} .628(\mathrm{I} .029-2.53 \mathrm{I})$ & 0.089 & I.39I (I.36I-2.355) & 0.101 \\
\hline
\end{tabular}

Abbreviations: $\mathrm{Cl}$, confidence interval; DFS, disease-free survival; $\Delta \mathrm{LMR}$, change in lymphocyte-to-monocyte ratio; HR, hazard ratio; NLR, neutrophil-to-lymphocyte ratio; $\triangle N L R$, change in NLR; NSCLC, non-small cell lung cancer; OS, overall survival. 

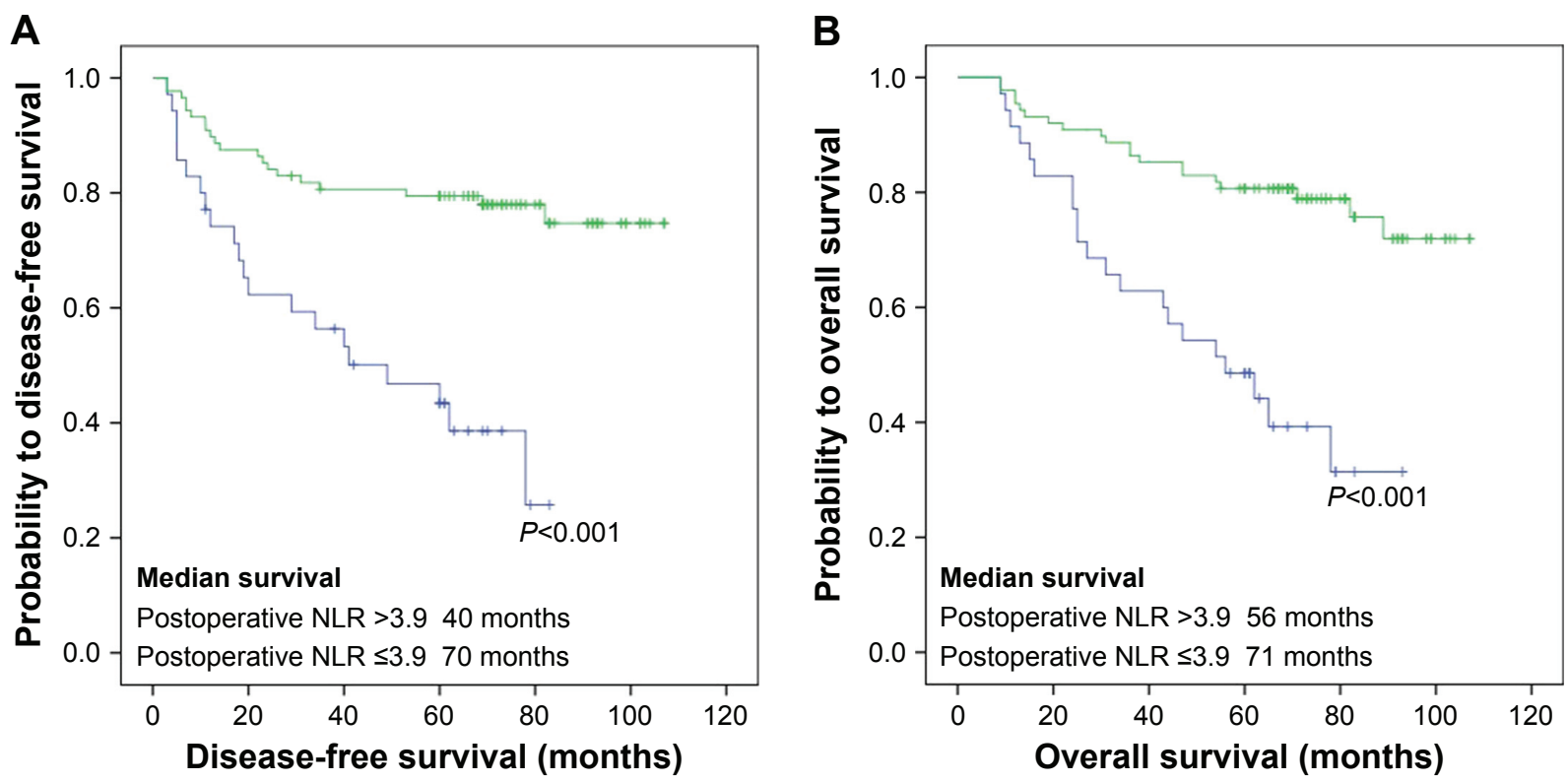

Postoperative NLR

$\neg>3.9-\neg \leq 3.9+>3.9$-censored $+\leq 3.9$-censored

Figure 2 Kaplan-Meier curves for disease-free survival (A) and overall survival (B) probability according to postoperative NLR level. Abbreviation: NLR, neutrophil-to-lymphocyte ratio.

prognostic markers for patients with NSCLC. ${ }^{8,19,20}$ However, these studies focused on the pretreatment values of inflammatory markers. Few studies have evaluated dynamic changes in markers, which might reflect the balance between the host inflammatory and immune responses. To our knowledge, this study is the first to demonstrate that postoperative NLR and $\triangle \mathrm{NLR}$ are independent prognostic factors for patients who have undergone complete resection of stage I NSCLC.

Based on individual data from 123 patients who underwent pulmonary resection of stage I NSCLC, our
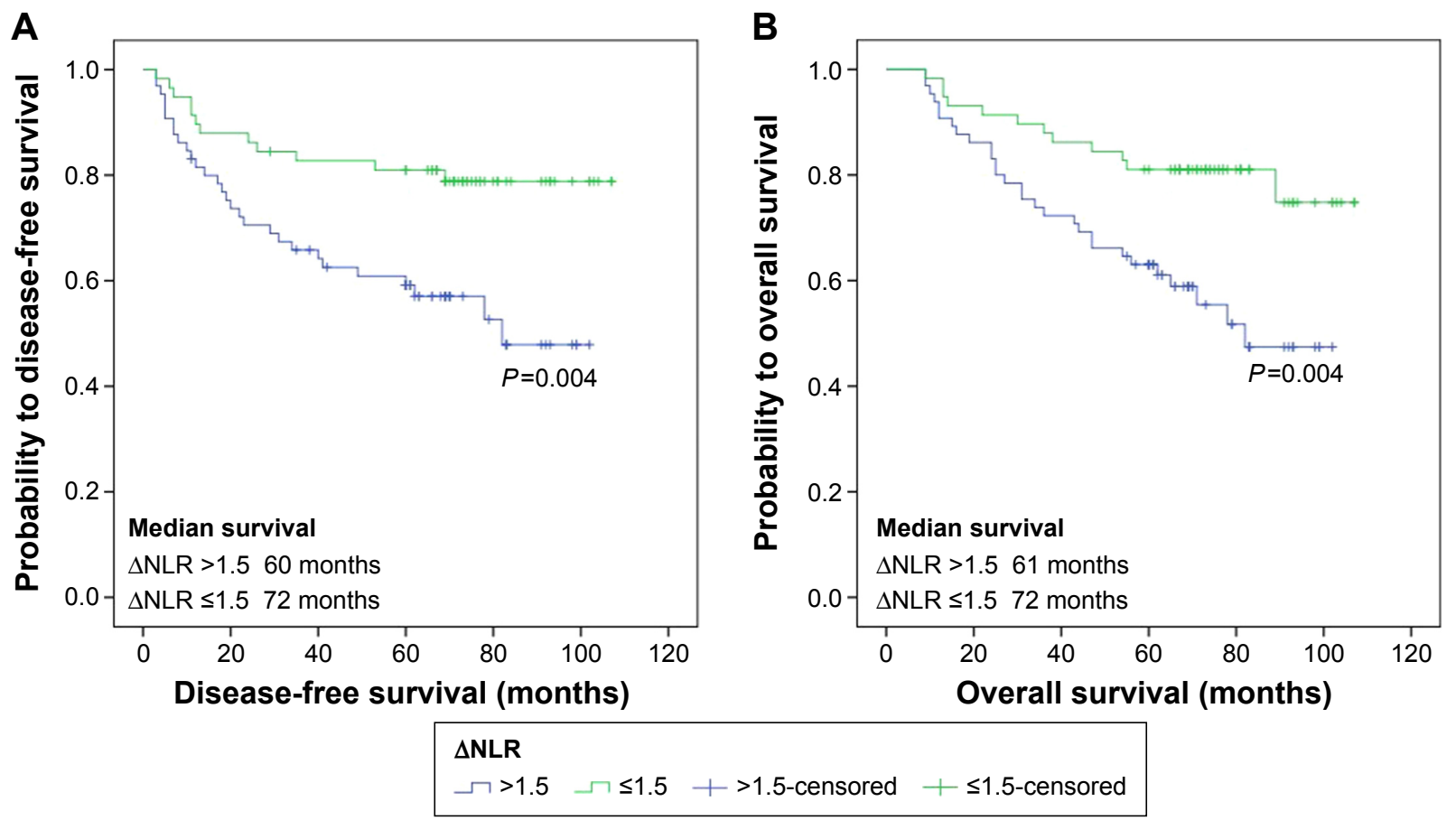

Figure 3 Kaplan-Meier curves for disease-free survival (A) and overall survival (B) probability according to $\triangle N L R$ level. Abbreviation: $\triangle \mathrm{NLR}$, change in neutrophil-to-lymphocyte ratio. 
retrospective analysis demonstrated that postoperative NLR and $\triangle \mathrm{NLR}$ - but not preoperative NLR - were significant prognostic factors. In recent years, some meta-analyses have supported the prognostic value of preoperative NLR in lung cancer. ${ }^{4,21,22}$ However, these studies enrolled patients with different stages of disease, especially patients with small-cell lung cancer, the biological behavior and prognosis of which differ from those of NSCLC. In comparison, few studies have focused on stage I NSCLC. Recently, a study by Miyazaki et $\mathrm{al}^{10}$ found that preoperative NLR was not correlated with 5-year survival in patients with stage I NSCLC. Accordingly, the understanding of preoperative NLR's predictive role has changed for patients undergoing complete resection of stage I NSCLC. This study results revealed that preoperative NLR was not a predictor of survival in stage I NSCLC, in agreement with the report by Miyazaki et al. To the best of our knowledge, there has been no related study on postoperative NLR or $\triangle N L R$ in patients with stage I NSCLC. This study confirmed that postoperative NLR $(\mathrm{HR}=2.435, P=0.001)$ and $\triangle \mathrm{NLR}(\mathrm{HR}=2.103, P=0.012)$ were independent risk factors that predicted DFS. Furthermore, our results confirm that the significant predictors of OS include postoperative NLR (HR $=2.747, P=0.001)$ and $\Delta \mathrm{NLR}(\mathrm{HR}=2.052, P=0.018)$. The results revealed that postoperative NLR and $\triangle$ NLR reflect the postoperative balance or change in the systematic inflammatory response from the preoperative period to the postoperative period, which may provide a more precise reflection of prognosis.

The relationship between NLR and prognosis currently appears to be complex, and the precise mechanisms involved are not fully understood. Increasing evidence supports the involvement of systemic inflammation in the extent of tumor progression and metastasis, ${ }^{23}$ with inflammatory processes contributing to cancer initiation, promotion, progression, and invasion. ${ }^{24,25}$ Neutrophils, the dominant subset of inflammatory cells, may stimulate tumor angiogenesis by producing vascular endothelial growth factor, matrix metalloproteinases, and elastases. ${ }^{26}$ On the other hand, lymphocytes in the tumor microenvironment play a substantial role in immune surveillance against tumor development. ${ }^{27}$ Hiraoka et $\mathrm{al}^{28}$ found that tumor infiltrating lymphocytes could inhibit tumor growth and were correlated with a favorable prognosis in lung cancer. Lymphocytes in the tumor microenvironment are mainly derived from the lymphocyte migration of peripheral blood, ${ }^{29,30}$ and the number of lymphocytes in the tumor microenvironment and in peripheral blood is considered as an important prognostic and predictive marker. ${ }^{31,32}$ Therefore, NLR could act as a marker reflecting the balance between host protumor inflammatory status and antitumor immune status. However, preoperative NLR only reflects the balance before surgery, while postoperative NLR represents the status after surgical removal of the tumor, which would be expected to provide a more precise indication of treatment response. Moreover, $\triangle \mathrm{NLR}$ represents the dynamic variation between host inflammatory response and immune response from the preoperative period to the postoperative period, which can be used for early evaluations of treatment efficacy and predictions of survival. ${ }^{15}$

The multivariate analysis in this study did not validate the associations between LMR, PLR, and long-term outcomes in patients undergoing complete resection of stage I NSCLC. LMR, one of the inflammatory biomarkers, was regarded as an independent prognostic factor for DFS and OS in previously untreated metastatic NSCLC patients receiving platinum-based doublet chemotherapy. ${ }^{33}$ However, Song et $\mathrm{al}^{34}$ found that the LMR values at initial diagnosis and disease progression were similar in patients with early-stage NSCLC $(P=0.287)$, which suggested that LMR would not be predictive of DFS. In a previous study of patients with all stages of NSCLC, PLR was observed to be an independent prognostic factor. ${ }^{20}$ However, for early-stage NSCLC, some studies confirmed that PLR was not associated with survival. ${ }^{5,6,10}$ Our results are in agreement with the latter studies, owing to the similar stages that were analyzed.

There has been no consistent opinion regarding the appropriate timing for postoperative measurement. Choi et $\mathrm{al}^{35}$ defined the postoperative NLR as the value obtained on postoperative day 1 . However, NLR shows a peak at 24 hours after surgery, ${ }^{36}$ which is probably influenced by psychological and physiological factors. For example, surgery is a source of stress for patients and could change the systemic inflammatory state. ${ }^{11}$ Because the literature has shown that inflammation due to the surgical wound healing process ceases 1 month after the operation, ${ }^{37}$ postoperative measurements were chosen to perform at least 1 month after surgery to minimize the disturbances caused by the operation.

Our study has several limitations. 1) It was a retrospective study with a small sample size, which might have contributed to selection bias. 2) Because sufficient information was unavailable, the effects of different treatment regimens on survival was not analyzed after recurrence. 3) The generalizability of our findings might be limited because of the single-center design of our study. Therefore, a prospective, large sample, multicenter clinical study is needed to verify the reported results. Finally, this study did not involve laboratory data of intraindividual variations (different collection times) 
and interindividual variations (different intervals, gender, or age), which might be the future research direction.

\section{Conclusion}

To the best of our knowledge, this is the first study to evaluate the prognostic significance of postoperative NLR and $\triangle \mathrm{NLR}$ in patients undergoing complete resection of stage I NSCLC. Postoperative NLR and $\triangle$ NLR - but not preoperative NLR - were independent prognostic factors for DFS and OS in these patients with stage I NSCLC. The patients will be classified into two groups as low risk and high risk for individual assessment according to different values of the two parameters in further work. Further investigations are warranted to validate our findings.

\section{Disclosure}

The authors report no conflicts of interest in this work.

\section{References}

1. Siegel RL, Miller KD, Jemal A. Cancer statistics, 2015. CA Cancer J Clin. 2015;65(1):5-29.

2. Siegel R, DeSantis C, Virgo K, et al. Cancer treatment and survivorship statistics, 2012. CA Cancer J Clin. 2012;62(4):220-241.

3. Brock MV, Hooker CM, Ota-Machida E, et al. DNA methylation markers and early recurrence in stage I lung cancer. $N$ Engl J Med. 2008;358(11): $1118-1128$.

4. Gu XB, Tian T, Tian XJ, Zhang XJ. Prognostic significance of neutrophil-to-lymphocyte ratio in non-small cell lung cancer: a metaanalysis. Sci Rep. 2015;5:12493.

5. Zhang T, Jiang Y, Qu X, Shen H, Liu Q, Du J. Evaluation of preoperative hematologic markers as prognostic factors and establishment of novel risk stratification in resected $\mathrm{pN} 0$ non-small-cell lung cancer. PLoS One. 2014;9(10):e111494.

6. Pinato DJ, Shiner RJ, Seckl MJ, Stebbing J, Sharma R, Mauri FA. Prognostic performance of inflammation-based prognostic indices in primary operable non-small cell lung cancer. Br J Cancer. 2014;110(8): 1930-1935.

7. Takahashi $\mathrm{Y}$, Horio $\mathrm{H}$, Hato $\mathrm{T}$, et al. Prognostic significance of preoperative neutrophil-lymphocyte ratios in patients with stage 1 non-small cell lung cancer after complete resection. Ann Surg Oncol. 2015;22(suppl 3): 1324-1331.

8. Zhang H, Xia H, Zhang L, Zhang B, Yue D, Wang C. Clinical significance of preoperative neutrophil-lymphocyte vs platelet-lymphocyte ratio in primary operable patients with non-small cell lung cancer. $\mathrm{Am}$ J Surg. 2015;210(3):526-535.

9. Liao Y, Ni Y, He R, et al. Clinical implications of fibroblast activation protein-alpha in non-small cell lung cancer after curative resection: a new predictor for prognosis. J Cancer Res Clin Oncol. 2013;139(9): $1523-1528$.

10. Miyazaki T, Yamasaki N, Tsuchiya T, et al. Inflammation-based scoring is a useful prognostic predictor of pulmonary resection for elderly patients with clinical stage I non-small-cell lung cancer. Eur J Cardiothorac Surg. 2015;47(4):e140-e145.

11. Li C, Wen TF, Yan LN, et al. Postoperative neutrophil-to-lymphocyte ratio plus platelet-to-lymphocyte ratio predicts the outcomes of hepatocellular carcinoma. $J$ Surg Res. 2015;198(1):73-79.

12. Shibutani M, Maeda K, Nagahara H, et al. The prognostic significance of a postoperative systemic inflammatory response in patients with colorectal cancer. World J Surg Oncol. 2015;13:194.
13. Kang M, Jeong CW, Kwak C, Kim HH, Ku JH. The prognostic significance of the early postoperative neutrophil-to-lymphocyte ratio in patients with urothelial carcinoma of the bladder undergoing radical cystectomy. Ann Surg Oncol. 2016;23(1):335-342.

14. Peng W, Li C, Wen TF, et al. Neutrophil to lymphocyte ratio changes predict small hepatocellular carcinoma survival. J Surg Res. 2014;192(2):402-408.

15. Dan J, Zhang Y, Peng Z, et al. Postoperative neutrophil-to-lymphocyte ratio change predicts survival of patients with small hepatocellular carcinoma undergoing radiofrequency ablation. PLoS One. 2013;8(3):e58184.

16. Detterbeck FC, Boffa DJ, Tanoue LT. The new lung cancer staging system. Chest. 2009;136(1):260-271.

17. Brambilla E, Travis WD, Colby TV, Corrin B, Shimosato Y. The new World Health Organization classification of lung tumours. Eur Respir J. 2001;18(6):1059-1068

18. Takahashi Y, Kawamura M, Hato T, Harada M, Matsutani N, Horio H. Neutrophil-lymphocyte ratio as a prognostic marker for lung adenocarcinoma after complete resection. World J Surg. 2016;40(2):365-372.

19. Hu P, Shen H, Wang G, Zhang P, Liu Q, Du J. Prognostic significance of systemic inflammation-based lymphocyte- monocyte ratio in patients with lung cancer: based on a large cohort study. PLoS One. 2014;9(9): e108062.

20. Kos M, Hocazade C, Kos FT, et al. Prognostic role of pretreatment platelet/lymphocyte ratio in patients with non-small cell lung cancer. Wien Klin Wochenschr. 2016;128(17-18):635-640.

21. Zhao QT, Yang Y, Xu S, et al. Prognostic role of neutrophil to lymphocyte ratio in lung cancers: a meta-analysis including 7,054 patients. Onco Targets Ther. 2015;8:2731-2738.

22. Yin $Y$, Wang J, Wang X, et al. Prognostic value of the neutrophil to lymphocyte ratio in lung cancer: a meta-analysis. Clinics (Sao Paulo). 2015;70(7):524-530.

23. Kundu JK, Surh YJ. Inflammation: gearing the journey to cancer. Mutat Res. 2008;659(1-2):15-30.

24. Grivennikov SI, Greten FR, Karin M. Immunity, inflammation, and cancer. Cell. 2010;140(6):883-899.

25. Schreiber RD, Old LJ, Smyth MJ. Cancer immunoediting: integrating immunity's roles in cancer suppression and promotion. Science. 2011;331(6024):1565-1570.

26. Vendramini-Costa DB, Carvalho JE. Molecular link mechanisms between inflammation and cancer. Curr Pharm Des. 2012;18(26):3831-3852.

27. Dunn GP, Old LJ, Schreiber RD. The immunobiology of cancer immunosurveillance and immunoediting. Immunity. 2004;21(2):137-148.

28. Hiraoka K, Miyamoto M, Cho Y, et al. Concurrent infiltration by CD8+ $\mathrm{T}$ cells and CD4+ $\mathrm{T}$ cells is a favourable prognostic factor in non-smallcell lung carcinoma. Br J Cancer. 2006;94(2):275-280.

29. Applegate KG, Balch CM, Pellis NR. In vitro migration of lymphocytes through collagen matrix: arrested locomotion in tumor-infiltrating lymphocytes. Cancer Res. 1990;50(22):7153-7158.

30. Ratner S, Heppner GH. Mechanisms of lymphocyte traffic in neoplasia. Anticancer Res. 1986;6(3 pt B):475-482.

31. Gutkin DW, Shurin MR. Clinical evaluation of systemic and local immune responses in cancer: time for integration. Cancer Immunol Immunother. 2014;63(1):45-57.

32. Galon J, Pages F, Marincola FM, et al. The immune score as a new possible approach for the classification of cancer. J Transl Med. 2012; 10:1.

33. Lin GN, Peng JW, Xiao JJ, Liu DY, Xia ZJ. Prognostic impact of circulating monocytes and lymphocyte-to-monocyte ratio on previously untreated metastatic non-small cell lung cancer patients receiving platinum-based doublet. Med Oncol. 2014;31(7):70.

34. Song YJ, Wang LX, Hong YQ, et al. Lymphocyte to monocyte ratio is associated with response to first-line platinum-based chemotherapy and prognosis of early-stage non-small cell lung cancer patients. Tumour Biol. 2016;37(4):5285-5293.

35. Choi JE, Villarreal J, Lasala J, et al. Perioperative neutrophil:lymphocyte ratio and postoperative NSAID use as predictors of survival after lung cancer surgery: a retrospective study. Cancer Med. 2015;4(6):825-833. 
36. Medina Fernandez FJ, Munoz-Casares FC, Arjona-Sanchez A, et al. Postoperative time course and utility of inflammatory markers in patients with ovarian peritoneal carcinomatosis treated with neoadjuvant chemotherapy, cytoreductive surgery, and HIPEC. Ann Surg Oncol. 2015;22(4):1332-1340.
37. Josa V, Krzystanek M, Eklund AC, et al. Relationship of postoperative thrombocytosis and survival of patients with colorectal cancer. Int J Surg. 2015;18:1-6.

\section{Publish your work in this journal}

OncoTargets and Therapy is an international, peer-reviewed, open access journal focusing on the pathological basis of all cancers, potential targets for therapy and treatment protocols employed to improve the management of cancer patients. The journal also focuses on the impact of management programs and new therapeutic agents and protocols on

\section{Dovepress}

patient perspectives such as quality of life, adherence and satisfaction. The manuscript management system is completely online and includes a very quick and fair peer-review system, which is all easy to use. Visit http://www.dovepress.com/testimonials.php to read real quotes from published authors.

Submit your manuscript here: http://www.dovepress.com/oncotargets-and-therapy-journal 